\title{
Preventative and therapeutic effects of a GABA transporter 1 inhibitor administered systemically in a mouse model of paclitaxel-induced neuropathic pain
}

\author{
Willias Masocha ${ }^{\text {Corresp., }}{ }^{1}$, Subramanian S Parvathy ${ }^{1}$ \\ ${ }^{1}$ Department of Pharmacology and Therapeutics, Faculty of Pharmacy, Kuwait University, Safat, Kuwait \\ Corresponding Author: Willias Masocha \\ Email address: masocha@hsc.edu.kw
}

Background: There is a dearth of drugs to manage a dose-limiting painful peripheral neuropathy induced by paclitaxel in some patients during the treatment of cancer. Gamma-aminobutyric acid transporter-1 (GAT-1) whose expression is increased in the brain and spinal cord during paclitaxelinduced neuropathic pain (PINP) might be a potential therapeutic target for managing PINP. Thus, our aim was to evaluate if systemic administration of a GAT-1 inhibitor ameliorates PINP.

Methods: The reaction latency to thermal stimuli (hot plate test; at $55^{\circ} \mathrm{C}$ ) and cold stimuli (cold plate test; at $4{ }^{\circ} \mathrm{C}$ ) of female BALB/C mice was recorded before and after intraperitoneal treatment with paclitaxel, its vehicle, and/or a selective GAT-1 inhibitor NO-711. The effects of NO-711 on motor coordination were evaluated using the rotarod test at a constant speed of 4 rpm or accelerating mode from 4 rpm to 40 rpm over 5 minutes.

Results: The coadministration of paclitaxel with N0-711 $3 \mathrm{mg} / \mathrm{kg}$ prevented the development of paclitaxel-induced thermal hyperalgesia and cold allodynia at day 7 after drug treatment. NO-711 at 3 $\mathrm{mg} / \mathrm{kg}$ produced antihyperalgesic activity up to 1 hour and antiallodynic activity up to 2 hours in mice with established paclitaxel-induced thermal hyperalgesia and cold allodynia. No motor deficits were observed with NO-711 at a dose of $3 \mathrm{mg} / \mathrm{kg}$, whereas a higher dose $5 \mathrm{mg} / \mathrm{kg}$ caused motor impairment and reduced mean time spent on the rotarod at a constant speed of $4 \mathrm{rpm}$. However, at a rotarod accelerating mode from $4 \mathrm{rpm}$ to $40 \mathrm{rpm}$ over 5 minutes, NO-711 $3 \mathrm{mg} / \mathrm{kg}$ caused motor impairment up to 1 hour, but had recovered by 2 hours.

Conclusions: These results show that systemic administration of the GAT-1 inhibitor NO-711 has preventative and therapeutic activity against paclitaxel-induced thermal hyperalgesia and cold allodynia. NO-711's antiallodynic effects, but not antihyperalgesic effects, were independent of its motor impairment/sedation properties. Thus, low doses of GAT-1 inhibitors could be useful for the prevention and treatment of PINP with proper dose titration to reduce motor impairment/sedation side effects. 
1 Preventative and therapeutic effects of a GABA transporter 1 inhibitor administered 2 systemically in a mouse model of paclitaxel-induced neuropathic pain

4 Willias Masocha*, Subramanian S. Parvathy

5 Department of Pharmacology and Therapeutics, Faculty of Pharmacy, Kuwait University, Safat,

6 Kuwait

7 Phone number: +965 24636078 Email: masocha@hsc.edu.kw

9 Abstract

Background: There is a dearth of drugs to manage a dose-limiting painful peripheral neuropathy induced by paclitaxel in some patients during the treatment of cancer. Gamma-aminobutyric acid transporter-1 (GAT-1) whose expression is increased in the brain and spinal cord during paclitaxel-induced neuropathic pain (PINP) might be a potential therapeutic target for managing PINP. Thus, our aim was to evaluate if systemic administration of a GAT-1 inhibitor ameliorates PINP.

Methods: The reaction latency to thermal stimuli (hot plate test; at $55^{\circ} \mathrm{C}$ ) and cold stimuli (cold plate test; at $4{ }^{\circ} \mathrm{C}$ ) of female BALB/c mice was recorded before and after intraperitoneal treatment with paclitaxel, its vehicle, and/or a selective GAT-1 inhibitor NO-711. The effects of NO-711 on motor coordination were evaluated using the rotarod test at a constant speed of $4 \mathrm{rpm}$ or accelerating mode from $4 \mathrm{rpm}$ to $40 \mathrm{rpm}$ over 5 minutes.

Results: The coadministration of paclitaxel with NO-711 $3 \mathrm{mg} / \mathrm{kg}$ prevented the development of paclitaxel-induced thermal hyperalgesia and cold allodynia at day 7 after drug treatment. NO-

23711 at $3 \mathrm{mg} / \mathrm{kg}$ produced antihyperalgesic activity up to 1 hour and antiallodynic activity up to 2 
24 hours in mice with established paclitaxel-induced thermal hyperalgesia and cold allodynia. No

25 motor deficits were observed with NO-711 at a dose of $3 \mathrm{mg} / \mathrm{kg}$, whereas a higher dose $5 \mathrm{mg} / \mathrm{kg}$

26 caused motor impairment and reduced mean time spent on the rotarod at a constant speed of 4

$27 \mathrm{rpm}$. However, at a rotarod accelerating mode from $4 \mathrm{rpm}$ to $40 \mathrm{rpm}$ over 5 minutes,NO-711 3

$28 \mathrm{mg} / \mathrm{kg}$ caused motor impairment up to 1 hour, but had recovered by 2 hours.

29 Conclusions: These results show that systemic administration of the GAT-1 inhibitor NO-711

30 has preventative and therapeutic activity against paclitaxel-induced thermal hyperalgesia and

31 cold allodynia. NO-711's antiallodynic effects, but not antihyperalgesic effects, were

32 independent of its motor impairment/sedation properties. Thus, low doses of GAT-1 inhibitors

33 could be useful for the prevention and treatment of PINP with proper dose titration to reduce

34 motor impairment/sedation side effects. 


\section{Introduction}

38 Chemotherapy-induced neuropathic pain (CINP) limits the use of some chemotherapeutic drugs, such as paclitaxel, oxaliplatin and vincristine, in the management of various types of cancer. The incidence of chemotherapy-induced peripheral neuropathy in patients treated with some chemotherapeutic drugs is very high, for example in patients treated with paclitaxel it is around $70.8 \%(95 \% \mathrm{CI}=43.5-98.1)$ (Seretny et al. 2014). In another study it was found that $64 \%$ of patients experienced chemotherapy-induced peripheral neuropathy during paclitaxel treatment and $27 \%$ of these patients were diagnosed with neuropathic pain (Reyes-Gibby et al. 2009). Unfortunately, there is a dearth of drugs to prevent or manage this type of pain. Currently, only duloxetine has a moderate recommendation for the management of CINP, whilst other drugs used for other neuropathic pain conditions may be given because of the limited CINP treatment options (Hershman et al. 2014). Thus, studies on the pathophysiology of CINP and the development of new treatment options are essential.

Using an animal model of paclitaxel-induced neuropathic pain (PINP) we recently observed an increased expression of gamma-aminobutyric acid transporter 1 (GAT-1) transcripts in the anterior cingulate cortex (ACC) (Masocha 2015); which is an area involved in pain perception and modulation (Seminowicz et al. 2009; Steenland et al. 2006; Xie et al. 2009). GAT-1 is responsible for most of the GABA uptake from the synaptic cleft in the brain (Borden 1996;

55 Conti et al. 1998; Jensen et al. 2003). In the same year (2015) another group also reported increased expression of GAT-1 in the spinal cord of an animal model of PINP (Yadav et al.

57 2015). In addition, they observed that intrathecal injection of a GAT-1 inhibitor ameliorates 
58 PINP (Yadav et al. 2015). Thus, suggesting that GAT-1 plays an important role in the

59 pathophysiology of PINP and is a potential therapeutic target.

60 Tiagabine, a GAT-1 inhibitor, is used for the treatment of epilepsy as an add-on therapy in the

61 treatment of partial seizures (Bialer et al. 2007). It has various adverse effects of which the most

62 common include fatigue, dizziness, psychomotor slowing, ataxia, somnolence, insomnia,

63 nausea/GI upset and weight change (Bialer et al. 2007; Vossler et al. 2013). Tiagabine has also

64 been shown to have some beneficial effects for the treatment of neuropathic pain (Novak et al.

65 2001). In animal models of neuropathic pain NO-711, a GAT-1 inhibitor,has been reported to

66 have antiallodynic and antihyperalgesic activity (Daemen et al. 2008; Yadav et al. 2015). NO-

67711 is a selective and the most potent non-competitive inhibitor of GAT-1 that can cross the

68 blood-brain barrier (Borden et al. 1994; Hog et al. 2006; Soudijn \& van Wijngaarden 2000).

69 However, in one study it was suggested that the observed antinociceptive activity of the GAT-1

70 inhibitor tiagabine could be attributed to sedative and motor-impairing properties, as these

71 properties can produce false positive effects in some pain tests (Salat et al. 2015).

72 The aim of this study was to evaluate whether systemic administration of a GAT-1 inhibitor can

73 prevent the development of PINP and also if it has therapeutic effects against established PINP at

74 doses that do not impair motor activity. 


\section{Materials and Methods}

\section{Animals}

77 Animals used in this study were handled in compliance with the Kuwait University, Health

Sciences Center (HSC), Animal Resources Centre (ARC) guidelines and in compliance with

79 Directive 2010/63/EU of the European Parliament and of the Council on the protection of

80 animals used for scientific purposes. All animal experiments were approved by the Ethical

81 Committee for the use of Laboratory Animals in Teaching and in Research, HSC, Kuwait

82 University. Female BALB/c mice (8 to 12 weeks old; $20-30 \mathrm{~g} ; \mathrm{n}=247$ ) supplied by the ARC at

83 the HSC, Kuwait University were used in this study. The animals were kept in temperature

84 controlled $\left(24 \pm 1^{\circ} \mathrm{C}\right)$ rooms with food and water given ad libitum.

\section{Administration of paclitaxel to induce thermal hyperalgesia and cold allodynia}

86 Paclitaxel (Tocris, Bristol, UK) was dissolved in a solution made up of 50\% Cremophor EL and

$8750 \%$ absolute ethanol to a concentration of $6 \mathrm{mg} / \mathrm{ml}$ and then diluted in normal saline $(\mathrm{NaCl}$ $0.9 \%$ ), to a final concentration of $0.2 \mathrm{mg} / \mathrm{ml}$ just before administration. Vehicle or paclitaxel 2 $\mathrm{mg} / \mathrm{kg}$ were injected intraperitoneally (i.p.) for 5 consecutive days, the cumulative dose of

90 paclitaxel was $10 \mathrm{mg} / \mathrm{kg}$ (the paclitaxel administration schedule is depicted in Figure 1). This

91 paclitaxel treatment regimen produces painful neuropathy and thermal hyperalgesia in mice

92 (Nieto et al. 2008; Parvathy \& Masocha 2013). 
94 1-[2-[[(diphenylmethylene)imino]oxy]ethyl]-1,2,5,6-tetrahydro-3-pyridinecarboxylic

acid

95 hydrochloride (NO-711) (Sigma, St. Louis, MO, USA) was dissolved in normal saline and

96 administered to mice i.p. at a volume of $10 \mathrm{ml} / \mathrm{kg}$ body mass.

97 Two treatment regimens were used to treat paclitaxel-treated mice; the first was a 98 preventative/prophylactic treatment (Figure 1A) and the second a therapeutic treatment (Figure 1B). The doses of NO-711 were chosen based on those previously shown to have antinociceptive 100 activity in mice (Kubo et al. 2009).

101 For the preventative treatment NO-711 $3 \mathrm{mg} / \mathrm{kg}$ was co-administered daily with paclitaxel, as 102 described above, for 5 consecutive days (Figure 1A). The mice were assessed for the 103 development of thermal hyperalgesia and cold allodynia on day 7 and those that received 104 paclitaxel plus NO-711 were compared with the mice treated with the paclitaxel plus vehicle (for NO-711) only.

For the therapeutic treatment, NO-711 at doses of 1 and $3 \mathrm{mg} / \mathrm{kg}$, was administered once at 7 days after first administration of paclitaxel (Figure 1B), when mice had developed thermal hyperalgesia, as previously described (Parvathy \& Masocha 2013), and cold allodynia.

For the rotarod test NO-711 was administered once at doses of 3 and $5 \mathrm{mg} / \mathrm{kg}$ to naïve mice.

\section{Assessment of thermal nociception}

111 Reaction latencies to the hot-plate test were measured as described previously (Parvathy \&

112 Masocha 2013), before (baseline latency), at day 7 after first injection of paclitaxel alone or 113 together with NO-711 (preventative treatment), and at various times on day 7 starting at 30 114 minutes after treatment with NO-711 (therapeutic treatment). Briefly, mice were individually 
115 placed on a hot plate (Panlab SL, Barcelona, Spain) with the temperature adjusted to $55 \pm 1{ }^{\circ} \mathrm{C}$.

116 The time to the first sign of nociception, paw licking, flinching or jump response to avoid the

117 heat was recorded and the animal immediately removed from the hot plate. A cut-off period of

11820 seconds was maintained to avoid damage to the paws. The observer was blinded to the

119 treatment the animal received.

\section{Assessment of cold allodynia}

121 Reaction latencies to the cold-plate test were measured as described previously (Sanna et al.

122 2014), before (baseline latency), at day 7 after first injection of paclitaxel alone or together with

123 NO-711 (preventative treatment), and at various times on day 7 starting at 30 minutes after

124 treatment with NO-711 (therapeutic treatment). Briefly, mice were individually placed on a cold 125 plate (Panlab SL, Barcelona, Spain) with the temperature adjusted to $4 \pm 1{ }^{\circ} \mathrm{C}$. The time to it 126 took for the animal lick its paws was recorded and the animal immediately removed from the 127 cold plate. A cut-off period of 60 seconds was maintained to avoid damage to the paws. The 128 observer was blinded to the treatment the animal received.

\section{Assessment of motor coordination}

130 Motor coordination was evaluated using the rotarod apparatus (Panlab SL, Barcelona, Spain)

131 using two protocols. In the first protocol the rotation of the rod was set at a constant speed of 4

$132 \mathrm{rpm}$. In the second protocol an accelerating mode from $4 \mathrm{rpm}$ to $40 \mathrm{rpm}$ over 5 minutes was

133 used, as described previously ((Galante et al. 2009). All animals were trained for 3 days. On the 134 test day, mice received single injections of NO-711 3 and $5 \mathrm{mg} / \mathrm{kg}$ or its vehicle (normal saline)

135 before the test. The latency (in seconds) for the first fall was recorded at $30 \mathrm{~min}, 1 \mathrm{hr}$ and $2 \mathrm{hrs}$ 
136 after administration of NO-711. The cut-off time was set at $300 \mathrm{~s}$. The observer was blinded to

137 the treatment the animal received.

\section{Statistical analyses}

139 Statistical analyses were performed using one-way analysis of variance (ANOVA) followed by

140 Newman-Keuls post-tests or two-way repeated measures ANOVA followed by Bonferroni post-

141 tests using GraphPad Prism software (version 5.0). The differences were considered significant

142 at $\mathrm{p}<0.05$. The results in the text and figures are expressed as the means \pm S.E.M.

\section{Results}

\section{Paclitaxel-induced thermal hyperalgesia and cold allodynia}

145 Mice treated with paclitaxel developed thermal hyperalgesia i.e. had significant lower reaction

146 latency times (about $27 \%$ lower) to the hot plate $\left(55^{\circ} \mathrm{C}\right)$ on day 7 compared to vehicle-treated 147 mice, $7.1 \pm 0.3$ s versus $9.7 \pm 0.2$, respectively $(p<0.05$; Figure $2 \mathrm{~A}$ ), similar to what we 148 described previously (Parvathy \& Masocha 2013).

149 Mice treated with paclitaxel also developed cold allodynia i.e. had significant lower reaction 150 latency times (about $31 \%$ lower) to the cold plate $\left(4{ }^{\circ} \mathrm{C}\right)$ on day 7 compared to vehicle-treated 151 mice, $38.4 \pm 3.2$ s versus $55.7 \pm 2.2$, respectively $(\mathrm{p}<0.05$; Figure $2 \mathrm{~B})$. 
155 Mice treated with paclitaxel had significant lower reaction latency times to the hot plate $\left(55^{\circ} \mathrm{C}\right)$

156 on day 7 compared to vehicle-treated mice, $6.3 \pm 0.3$ s versus $9.5 \pm 0.4$, respectively $(p<0.05$;

157 Figure 3A). On the other hand, mice treated with paclitaxel plus NO-711 (3 mg/kg)

158 consecutively for 5 days had reaction latency times similar to the vehicle-only treated control

159 mice, $8.7 \pm 0.4 \mathrm{~s}$ versus $9.5 \pm 0.4$, respectively $(\mathrm{p}>0.05)$, which were significantly higher than

160 those of the mice treated with paclitaxel plus vehicle $(\mathrm{p}<0.01$; Figure $3 \mathrm{~A})$.

161 Mice treated with paclitaxel had significant lower reaction latency times to the cold plate $\left(4^{\circ} \mathrm{C}\right)$

162 on day 7 compared to vehicle-treated mice, $32.3 \pm 1.7$ s versus $58.7 \pm 0.4$, respectively $(\mathrm{p}<0.05$;

163 Figure 3B). On the other hand, mice treated with paclitaxel plus NO-711 (3 mg/kg)

164 consecutively for 5 days had reaction latency times similar to the vehicle-only treated control

165 mice, $51.2 \pm 3.3 \mathrm{~s}$ versus $59.7 \pm 0.3$, respectively $(\mathrm{p}>0.05)$, which were significantly higher than

166 those of the mice treated with paclitaxel plus vehicle $(p<0.01$; Figure $3 \mathrm{~A})$.

NO-711 alleviates established paclitaxel-induced thermal hyperalgesia and cold allodynia

169

170

171

172

173

174

175

176

Mice with paclitaxel-induced thermal hyperalgesia and cold allodynia (i.e. mice with significantly lower reaction times after treatment with paclitaxel compared to pretreatment values) were treated with two doses of NO-711, 1 and $3 \mathrm{mg} / \mathrm{kg}$.

The intraperitoneal administration of vehicle or a lower dose of NO-711 $(1 \mathrm{mg} / \mathrm{kg}) \mathrm{did}$ not change the reaction latency to thermal stimuli $\left(55^{\circ} \mathrm{C}\right)$ in mice with paclitaxel-induced thermal hyperalgesia compared to before vehicle or NO-711 $1 \mathrm{mg} / \mathrm{kg}$ administration at day $7(\mathrm{p}>0.05$; Figure 4A). However, NO-711 at a dose of $3 \mathrm{mg} / \mathrm{kg}$ produced significant increase in reaction latency in mice with paclitaxel-induced thermal hyperalgesia at 30 minutes and 1 hour post drug 
177 administration compared to mice treated with vehicle and before NO-711 administration at day 7

$178(\mathrm{p}<0.01)$ but had not effect at 2 hours hour post drug administration $(p>0.05$; Figure $4 \mathrm{~A})$. The

179 mice with paclitaxel-induced thermal hyperalgesia treated with NO-711 at a dose of $3 \mathrm{mg} / \mathrm{kg} \mathrm{had}$

180 reaction latency at 30 minutes and 1 hour that was not significantly different to the reaction

181 latency before paclitaxel administration to mice, $10.1 \pm 0.6 \mathrm{~s}, 8.8 \pm 0.6 \mathrm{~s}$ and $9.7 \pm 0.3 \mathrm{~s}$

182 respectively $(\mathrm{p}>0.05)$ but was significantly lower at 2 hours $7.8 \pm 0.4 \mathrm{~s}(\mathrm{p}<0.05$; Figure 4A).

183 The intraperitoneal administration of vehicle or a lower dose of NO-711 (1 mg/kg) did not 184 change the reaction latency to cols stimuli $\left(4^{\circ} \mathrm{C}\right)$ in mice with paclitaxel-induced cold allodynia 185 compared to before vehicle or NO-711 $1 \mathrm{mg} / \mathrm{kg}$ administration at day 7 ( $\mathrm{p}>0.05$; Figure 4B). 186 However, NO-711 at a dose of $3 \mathrm{mg} / \mathrm{kg}$ produced significant increase in reaction latency in mice 187 with paclitaxel-induced cold allodynia at all times measured 30 minutes, 1 hour and 2 hours, post 188 drug administration compared to mice treated with vehicle and before NO-711 administration at 189 day $7(\mathrm{p}<0.01$; Figure 4B). The mice with paclitaxel-induced thermal hyperalgesia treated with $190 \mathrm{NO}-711$ at a dose of $3 \mathrm{mg} / \mathrm{kg}$ had reaction latency at 30 minutes, 1 hour and 2 hours that was not 191 significantly different to the reaction latency before paclitaxel administration to mice, $60 \mathrm{~s}, 60 \mathrm{~s}$, $19256.2 \pm 3.6 \mathrm{~s}$ and $57.9 \pm 0.8 \mathrm{~s}$ respectively $(\mathrm{p}>0.05$; Figure $4 \mathrm{~B})$.

\section{Motor coordination}

Side effects of GAT-1 inhibitors such as tiagabine include sedation and impairment of motor coordination (Salat et al. 2015). Impairment of motor coordination and sedation affects the results of behavioural tests, including the hot plate test. Thus the effect of NO-711 on motor coordination was evaluated using the rotarod test. No significant differences of the mean time 
199 spent on the rotarod were observed between mice treated with vehicle $(300 \mathrm{~s})$ and the mice 200 treated with NO-711 $3 \mathrm{mg} / \mathrm{kg}(265 \pm 35 \mathrm{~s}, 269 \pm 30 \mathrm{~s}$ and $300 \mathrm{~s}$ at 30 minutes, 1 hour and 2 201 hours after drug administration, respectively ( $p>0.05$; Figure 5A) at a rotarod constant speed of $2024 \mathrm{rpm}$. However, mice treated with a higher dose of NO-711, $5 \mathrm{mg} / \mathrm{kg}$, had a significant decrease 203 in the mean time spent on the rotarod from $300 \mathrm{~s}$ to $123 \pm 52 \mathrm{~s}$ and $185 \pm 35 \mathrm{~s}$ at 30 minutes and 2041 hour after drug administration, respectively ( $\mathrm{p}<0.01$; Figure 5A), indicating motor 205 impairment, but had recovered by 2 hours, $300 \mathrm{~s}(\mathrm{p}>0.05)$. When NO-711 $3 \mathrm{mg} / \mathrm{kg}$ was 206 evaluated at a rotarod accelerating mode from $4 \mathrm{rpm}$ to $40 \mathrm{rpm}$ over 5 minutes, it had a 207 significant decrease in the mean time spent on the rotarod from $68.8 \pm 3.8 \mathrm{~s}$ to $23.8 \pm 5.4 \mathrm{~s}$ and $20848.6 \pm 4.3 \mathrm{~s}$ at 30 minutes and 1 hour after drug administration, respectively $(\mathrm{p}<0.01$; Figure 209 5B), indicating motor impairment, but had recovered by 2 hours, $65.9 \pm 3.6 \mathrm{~s}(\mathrm{p}>0.05)$. 


\section{Discussion}

212 The results of this study show that systemic administration of NO-711, a GAT-1 inhibitor,

213 prevents the development of paclitaxel-induced thermal hyperalgesia and cold allodynia and also

214 has antihyperalgesic and antiallodynic activity in mice with established paclitaxel-induced

215 thermal hyperalgesia and cold allodynia. However, the antihyperalgesic effects of NO-711 could

216 not be separated from its sedative and motor-impairing properties, whereas its antiallodynic

217 effects were independent of its sedative and motor-impairing properties.

218 GAT-1 inhibitors have anti-seizure activities and one of them, tiagabine, is used for the treatment

219 of epilepsy as an add-on therapy in the treatment of partial seizures (Bialer et al. 2007). They

220 have also been shown to have antinociceptive and antiallodynic activities (Daemen et al. 2008;

221 Ipponi et al. 1999; Li et al. 2011; Yadav et al. 2015). Subcutaneous administration of NO-711

222 produced antiallodynic activities in rats with CCI-induced neuropathic pain (Daemen et al.

223 2008). Intrathecal administration of NO-711 produced antiallodynic activities rats with CCI-

224 induced neuropathic pain and in rats with PINP (Li et al. 2011; Yadav et al. 2015). On the other

225 hand, intraperitoneal administration of another GAT-1 inhibitor SKF89976A did not have effect

226 on established allodynia in a partial sciatic nerve ligation (PSL) mouse model (Jinzenji et al.

227 2014). Intraperitoneal administration of tiagabine reversed SCI-induced neuropathic pain (

228 (Meisner et al. 2010). However, in one study it was suggested that the observed antinociceptive

229 activity of tiagabine could be attributed to sedative and motor-impairing properties, as these

230 properties can produce false positive effects in some pain tests (Salat et al. 2015). In the rotarod

231 test we observed that $\mathrm{NO}-711$ at a dose of $3 \mathrm{mg} / \mathrm{kg}$ did show significant motor impairment at

232 low speed $(4 \mathrm{rpm})$ on the rotarod but showed motor impairment/sedation up to 1 hour at an 233 accelerating mode $4-40 \mathrm{rpm}$ ) of the rotarod, which is in contrast to what has been described 
234 before for that dose i.e. it did not cause motor/hypnotic effects (Kubo et al. 2009). A higher dose,

$2355 \mathrm{mg} / \mathrm{kg}$, caused motor impairment/sedation, even at low speed (4 rpm) on the rotarod, similar to

236 what has been observed with other high doses of NO-711 (Kubo et al. 2009; Suzdak et al. 1992).

237 Thus, NO-711 has a dose-dependent motor impairment/sedation effect as described previously

238 (Suzdak et al. 1992). In order to separate antinociceptive, antihyperalgesic or antiallodynic

239 effects of NO-711 from motor impairment/sedation effect a dose of $3 \mathrm{mg} / \mathrm{kg}$ was used for

240 evaluation of the activity of the compound against paclitaxel-induced thermal hyperalgesia and

241 cold allodynia, up to 2 hours, a time point when the drug had no motor impairment/sedation

242 effects.

243 Intrathecal administration of NO-711 has recently been shown to attenuate established paclitaxel

244 induced mechanical and thermal hyperalgesia in rats without causing motor impairment or

245 sedation (Yadav et al. 2015). In the current study systemic (intraperitoneal) administration of

$246 \mathrm{NO}-711$ at a dose of $3 \mathrm{mg} / \mathrm{kg}$ attenuated established paclitaxel-induced thermal hyperalgesia and

247 cold allodynia in mice. However, the effect of NO-711 on paclitaxel-induced thermal

248 hyperalgesia could not be separated from its no motor impairment/sedation effects because they

249 both lasted up to 1 hour after NO-711 administration. On the other hand, the effect of NO-711 on

250 paclitaxel-induced cold allodynia could be separated from its no motor impairment/sedation

251 effects because motor impairment lasted up to 1 hour after NO-711 administration, whereas the

252 drug had antiallodynic activity at 2 hours when it did not have motor impairment/sedation

253 effects. No other studies have reported the effects of a systemically administered GAT-1

254 inhibitor on PINP. However, systemic administration of GAT-1 inhibitors has been reported to

255 attenuate thermal hyperalgesia and allodynia in other models of neuropathic pain such as chronic

256 constriction injury of the sciatic nerve, spinal nerve ligation (Daemen et al. 2008; Giardina et al. 
257 1998). Thus, systemic administration of NO-711 has antiallodynic activities against PINP similar

258 to the systemic administration of another GAT-1 inhibitor, tiagabine, in other models of 259 neuropathic pain.

260 Our findings and those Yadav and colleagues showing increased GAT-1 expression in the CNS, 261 in the ACC and spinal cord, respectively, suggests an important role of GAT-1 in the 262 pathophysiology of PINP (Masocha 2015; Yadav et al. 2015). Thus, we explored whether 263 preventative treatment by inhibiting GAT-1 activity could be potentially useful to prevent the 264 development of PINP. Our findings show that coadministration of NO-711 at a dose of $3 \mathrm{mg} / \mathrm{kg}$ 265 with paclitaxel for five consecutive days prevented the development of paclitaxel-induced 266 thermal hyperalgesia and cold allodynia. Thus, indicating that increased GAT-1 expression and 267 activity play a role in the development of PINP. The possible mechanism of GAT-1 inhibitors in 268 PINP is possibly through restoration of ambient GABA levels. The increased GAT-1 expression 269 during PINP consequently increases GABA uptake and thus reduces ambient GABA levels and 270 decreases inhibitory tone. Recently, we reported that there is increased excitability in the ACC 271 because of a deficiency in GABA-mediated neurotransmission in that brain area and addition of 272 exogenous GABA reverses the increased excitability in the ACC induced by paclitaxel (Nashawi 273 et al. 2016).

\section{Conclusions}

276 In conclusion our results show that systemic administration of a GAT-1 inhibitor, NO-711,

277 prevents the development of paclitaxel-induced thermal hyperalgesia and cold allodynia and 278 alleviates established paclitaxel-induced thermal hyperalgesia and cold allodynia. The 
279 antiallodynic activity of the GAT-1 inhibitor is independent of its motor impairment/sedative 280 activities, whereas its antihyperalgesic activity are not. Thus, low doses of GAT-1 inhibitors 281 have potential therapeutic activity to prevent or manage PINP and CINP in general with proper 282 dose titration to reduce motor impairment/sedation side effects. Therefore the possible clinical 283 use of GAT-1 inhibitors, which are already in the clinics such as tiagabine, against CINP 284 warrants further research. 


\section{Acknowledgements}

287 We are grateful to Ms Amal Thomas for her technical assistance and the staff from the Animal

288 Resources Centre, HSC, Kuwait University for their support. 
291

292

293

294

295

296

297

298

299

300

301

302

303

304

305

306

307

308

309

310

311

312

313

314

315

316

317

318

319

320

321

322

323

324

325

326

327

328

329

330

331

332

333

334

335

Bialer M, Johannessen SI, Kupferberg HJ, Levy RH, Perucca E, and Tomson T. 2007. Progress report on new antiepileptic drugs: a summary of the Eigth Eilat Conference (EILAT VIII). Epilepsy Res 73:152.

Borden LA. 1996. GABA transporter heterogeneity: pharmacology and cellular localization. Neurochem Int 29:335-356.

Borden LA, Murali Dhar TG, Smith KE, Weinshank RL, Branchek TA, and Gluchowski C. 1994. Tiagabine, SK\&F 89976-A, Cl-966, and NNC-711 are selective for the cloned GABA transporter GAT-1. Eur J Pharmacol 269:219-224.

Conti F, Melone M, De Biasi S, Minelli A, Brecha NC, and Ducati A. 1998. Neuronal and glial localization of GAT-1, a high-affinity gamma-aminobutyric acid plasma membrane transporter, in human cerebral cortex: with a note on its distribution in monkey cortex. J Comp Neurol 396:51-63.

Daemen MA, Hoogland G, Cijntje JM, and Spincemaille GH. 2008. Upregulation of the GABA-transporter GAT-1 in the spinal cord contributes to pain behaviour in experimental neuropathy. Neurosci Lett 444:112-115.

Galante M, Jani H, Vanes L, Daniel H, Fisher EM, Tybulewicz VL, Bliss TV, and Morice E. 2009. Impairments in motor coordination without major changes in cerebellar plasticity in the Tc1 mouse model of Down syndrome. Hum Mol Genet 18:1449-1463.

Giardina WJ, Decker MW, Porsolt RD, Roux S, Collins SD, Kim DJB, and Bannon AW. 1998. An evaluation of the GABA uptake blocker tiagabine in animal models of neuropathic and nociceptive pain. Drug Development Res 44:106-103.

Hershman DL, Lacchetti C, Dworkin RH, Lavoie Smith EM, Bleeker J, Cavaletti G, Chauhan C, Gavin P, Lavino A, Lustberg MB, Paice J, Schneider B, Smith ML, Smith T, Terstriep S, Wagner-Johnston N, Bak K, Loprinzi CL, and American Society of Clinical O. 2014. Prevention and management of chemotherapy-induced peripheral neuropathy in survivors of adult cancers: American Society of Clinical Oncology clinical practice guideline. J Clin Oncol 32:1941-1967.

Hog S, Greenwood JR, Madsen KB, Larsson OM, Frolund B, Schousboe A, Krogsgaard-Larsen P, and Clausen RP. 2006. Structure-activity relationships of selective GABA uptake inhibitors. Curr Top Med Chem 6:1861-1882.

Ipponi A, Lamberti C, Medica A, Bartolini A, and Malmberg-Aiello P. 1999. Tiagabine antinociception in rodents depends on $\mathrm{GABA}(\mathrm{B})$ receptor activation: parallel antinociception testing and medial thalamus GABA microdialysis. Eur J Pharmacol 368:205-211.

Jensen K, Chiu CS, Sokolova I, Lester HA, and Mody I. 2003. GABA transporter-1 (GAT1)-deficient mice: differential tonic activation of GABAA versus GABAB receptors in the hippocampus. J Neurophysiol 90:2690-2701.

Jinzenji A, Sogawa C, Miyawaki T, Wen XF, Yi D, Ohyama K, Kitayama S, Sogawa N, and Morita K. 2014. Antiallodynic action of 1-(3-(9H-Carbazol-9-yl)-1-propyl)-4-(2-methyoxyphenyl)-4-piperidinol (NNC05-2090), a betaine/GABA transporter inhibitor. J Pharmacol Sci 125:217-226.

Kubo K, Nishikawa K, Ishizeki J, Hardy-Yamada M, Yanagawa Y, and Saito S. 2009. Thermal hyperalgesia via supraspinal mechanisms in mice lacking glutamate decarboxylase 65. J Pharmacol Exp Ther 331:162-169.

Li Y, Li Y, Gu P, Fu B, Liu F, and Li E. 2011. Analgesic effect of intrathecally gamma-aminobutyric acid transporter-1 inhibitor NO-711 administrating on neuropathic pain in rats. Neurosci Lett 494:6-9.

Masocha W. 2015. Comprehensive analysis of the GABAergic system gene expression profile in the anterior cingulate cortex of mice with Paclitaxel-induced neuropathic pain. Gene Expr 16:145153. 
Meisner JG, Marsh AD, and Marsh DR. 2010. Loss of GABAergic interneurons in laminae I-III of the spinal cord dorsal horn contributes to reduced GABAergic tone and neuropathic pain after spinal cord injury. J Neurotrauma 27:729-737.

Nashawi H, Masocha W, Edafiogho IO, and Kombian SB. 2016. Paclitaxel Causes Electrophysiological Changes in the Anterior Cingulate Cortex via Modulation of the gamma-Aminobutyric Acid-ergic System. Med Princ Pract 25:423-428.

Nieto FR, Entrena JM, Cendan CM, Pozo ED, Vela JM, and Baeyens JM. 2008. Tetrodotoxin inhibits the development and expression of neuropathic pain induced by paclitaxel in mice. Pain 137:520531.

Novak V, Kanard R, Kissel JT, and Mendell JR. 2001. Treatment of painful sensory neuropathy with tiagabine: a pilot study. Clin Auton Res 11:357-361.

Parvathy SS, and Masocha W. 2013. Matrix metalloproteinase inhibitor COL-3 prevents the development of paclitaxel-induced hyperalgesia in mice. Med Princ Pract 22:35-41.

Reyes-Gibby CC, Morrow PK, Buzdar A, and Shete S. 2009. Chemotherapy-induced peripheral neuropathy as a predictor of neuropathic pain in breast cancer patients previously treated with paclitaxel. J Pain 10:1146-1150.

Salat K, Podkowa A, Kowalczyk P, Kulig K, Dziubina A, Filipek B, and Librowski T. 2015. Anticonvulsant active inhibitor of GABA transporter subtype 1, tiagabine, with activity in mouse models of anxiety, pain and depression. Pharmacol Rep 67:465-472.

Sanna MD, Quattrone A, Ghelardini C, and Galeotti N. 2014. PKC-mediated HuD-GAP43 pathway activation in a mouse model of antiretroviral painful neuropathy. Pharmacol Res 81:44-53.

Seminowicz DA, Laferriere AL, Millecamps M, Yu JS, Coderre TJ, and Bushnell MC. 2009. MRI structural brain changes associated with sensory and emotional function in a rat model of long-term neuropathic pain. Neuroimage 47:1007-1014.

Seretny M, Currie GL, Sena ES, Ramnarine S, Grant R, MacLeod MR, Colvin LA, and Fallon M. 2014. Incidence, prevalence, and predictors of chemotherapy-induced peripheral neuropathy: A systematic review and meta-analysis. Pain 155:2461-2470.

Soudijn W, and van Wijngaarden I. 2000. The GABA transporter and its inhibitors. Curr Med Chem 7:1063-1079.

Steenland HW, Ko SW, Wu L, and Zhuo M. 2006. Hot receptors in the brain. Mol Pain 2:34.

Suzdak PD, Frederiksen K, Andersen KE, Sorensen PO, Knutsen LJ, and Nielsen EB. 1992. NNC-711, a novel potent and selective gamma-aminobutyric acid uptake inhibitor: pharmacological characterization. Eur J Pharmacol 224:189-198.

Vossler DG, Morris GL, 3rd, Harden CL, Montouris G, Faught E, Kanner AM, Fix A, French JA, and Postmarketing Antiepileptic Drug Survey group study i. 2013. Tiagabine in clinical practice: effects on seizure control and behavior. Epilepsy Behav 28:211-216.

Xie YF, Huo FQ, and Tang JS. 2009. Cerebral cortex modulation of pain. Acta Pharmacol Sin 30:31-41.

Yadav R, Yan X, Maixner DW, Gao M, and Weng HR. 2015. Blocking the GABA transporter GAT-1 ameliorates spinal GABAergic disinhibition and neuropathic pain induced by paclitaxel. J Neurochem 133:857-869. 


\section{Figure 1 (on next page)}

Drug administration schedule for preventative and therapeutic treatment with NO-711 against paclitaxel-induced thermal hyperalgesia.

(A) Treatment with NO-711 to prevent the development of paclitaxel-induced thermal hyperalgesia (B) Therapeutic treatment with NO-711 to alleviate established paclitaxelinduced thermal hyperalgesia. The arrows indicate the days when the drugs were intraperitoneally administered. 
PeerJ Manuscript to be reviewed

A $\quad$ Paclitaxel $2 \mathrm{mg} / \mathrm{kg}$

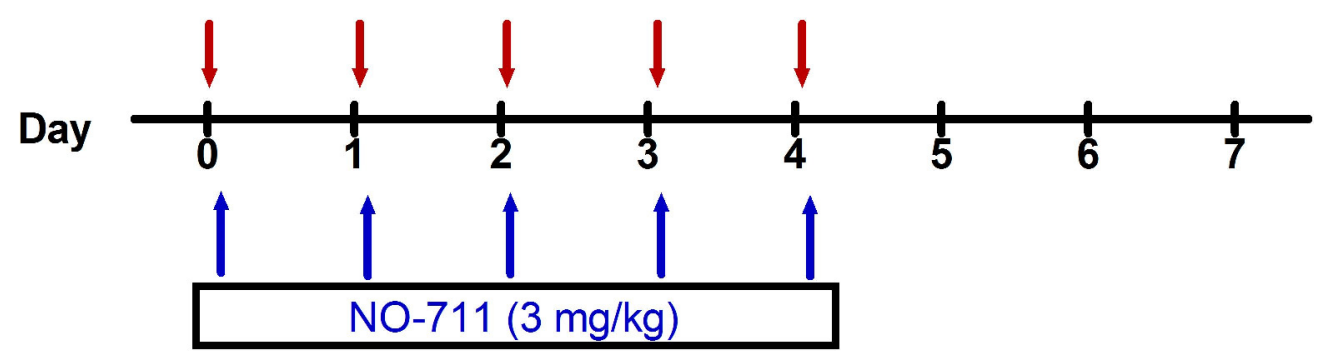

B
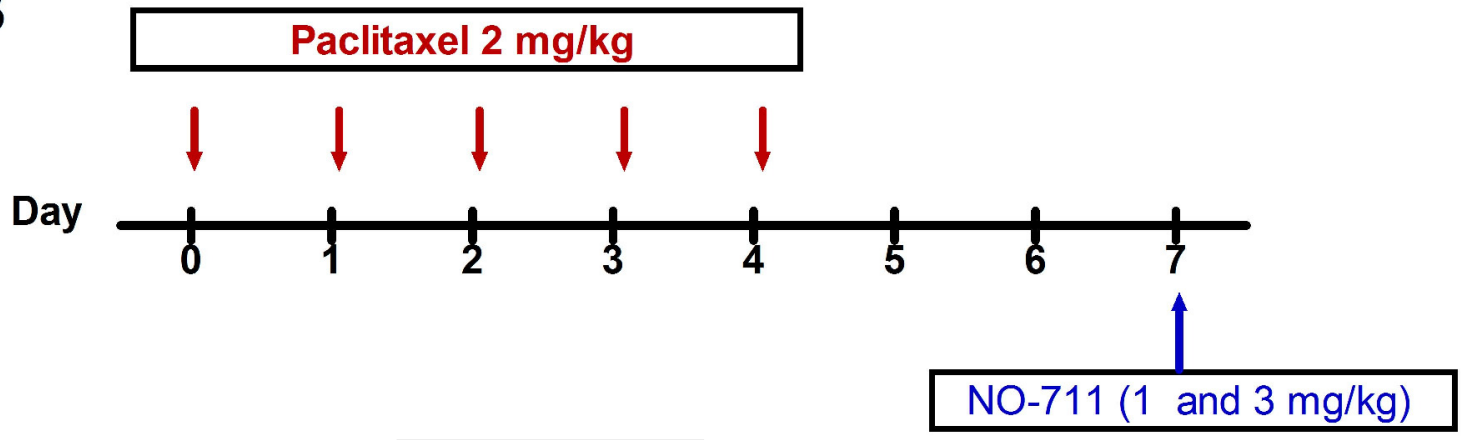


\section{Figure 2 (on next page)}

Paclitaxel-induced thermal hyperalgesia and cold allodynia in BALB/C mice.

(A) Thermal hyperalgesia in BALB/C mice at day 7 post first inoculation of paclitaxel in a hot plate test $\left(55^{\circ} \mathrm{C}\right)$. Each point represents the mean \pm S.E.M of values obtained from 15 vehicle-treated and 15 paclitaxel-treated animals. (B) Cold allodynia in BALB/c mice at day 7 post first inoculation of paclitaxel in a cold plate test $\left(4^{\circ} \mathrm{C}\right)$. Each point represents the mean \pm S.E.M of values obtained from 12 vehicle-treated and 13 paclitaxel-treated animals. $* * p<$ 0.01 compared to drug vehicle at the same day after treatment and \#\#p<0.01 compared to pretreatment (PRE) values (Student's $t$ test). 
PeerJ

A Thermal hyperalgesia

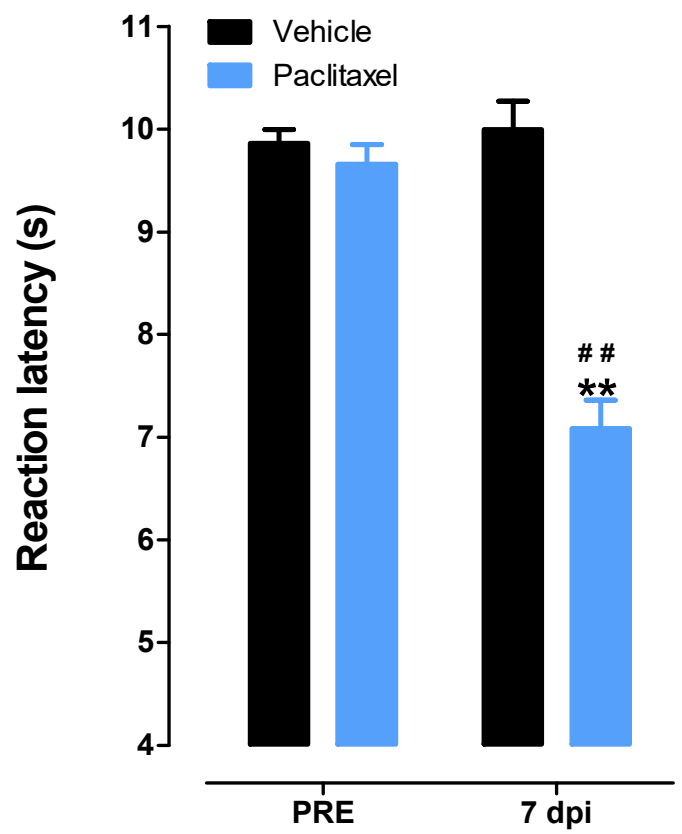

Manuscript to be reviewed

B Cold allodynia

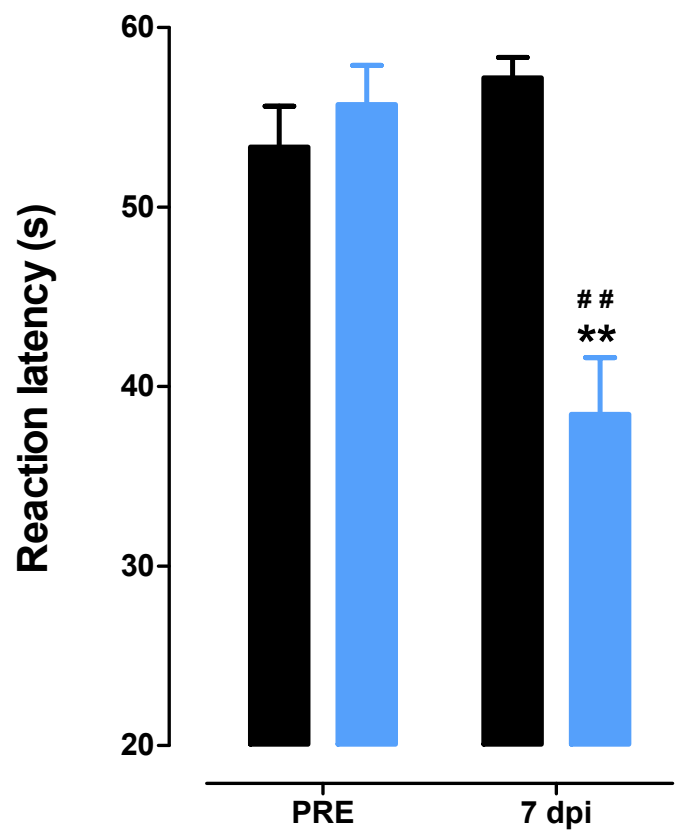


Figure 3 (on next page)

Coadministration of NO-711 with paclitaxel protects against the development of paclitaxel-induced thermal hyperalgesia and cold allodynia. 


\section{Thermal hyperalgesia}

Vehicles only

Paclitaxel + vehicle

A

Paclitaxel + NO-711 $3 \mathrm{mg} / \mathrm{kg}$

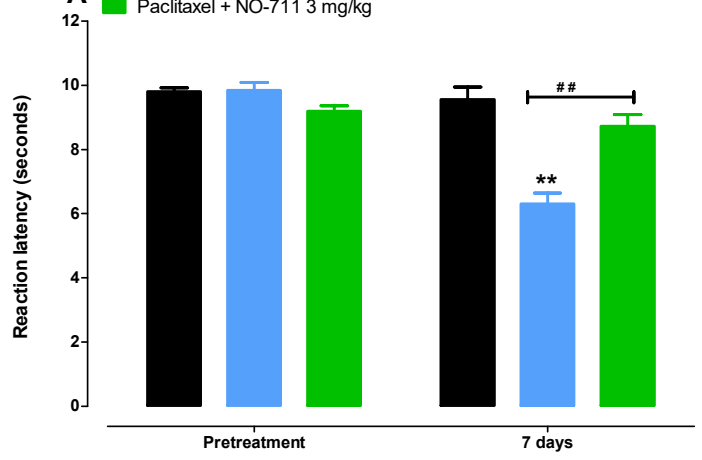

Time after first coadministration of drugs

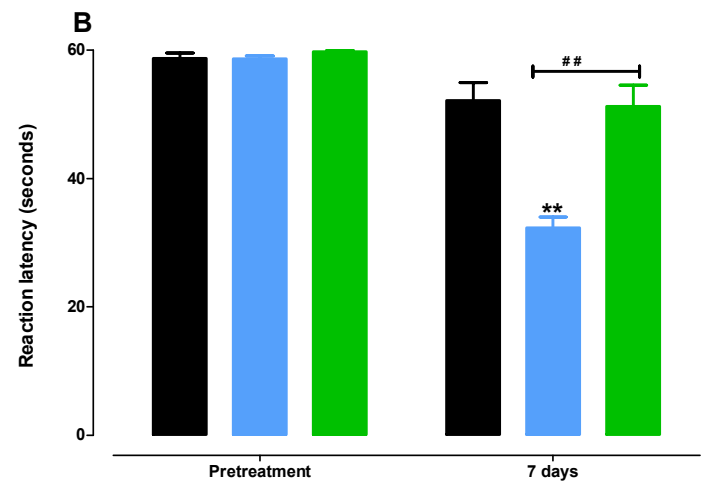

Time after first coadministration of drugs 


\section{Figure 4(on next page)}

Antihyperalgesic and antiallodynic effects of NO-711 on BALB/C mice with paclitaxelinduced thermal hyperalgesia and cold allodynia.

(A) Reaction latency times (taken before (PRE) and at day 7 post first administration of paclitaxel) at different times after treatment with NO-711 (1 and $3 \mathrm{mg} / \mathrm{kg}$ ) or its vehicle in a hot-plate test $\left(55^{\circ} \mathrm{C}\right)$. Each bar represents the mean \pm S.E.M of values obtained from 8-16 animals. (B) Reaction latency times (taken before (PRE) and at day 7 post first administration of paclitaxel) at different times after treatment with NO-711 (1 and $3 \mathrm{mg} / \mathrm{kg}$ ) or its vehicle in a cold-plate test $\left(55^{\circ} \mathrm{C}\right)$. Each bar represents the mean \pm S.E.M of values obtained from 5-7 animals. ${ }^{* *} p<0.01$ compared to drug vehicle at the same time point after treatment (twoway repeated measures ANOVA followed by Bonferroni test); $\# p<0.5, \# \# p<0.01$ compared to day 7 post paclitaxel values before NO-711 administration; and $\$ p<0.5$, \$\$ $p$ $<0.01$ compared to values before administration of paclitaxel (Pre-paclitaxel) (one-way ANOVA followed by Newman-Keuls post-test). 


\section{Thermal hyperalgesia}

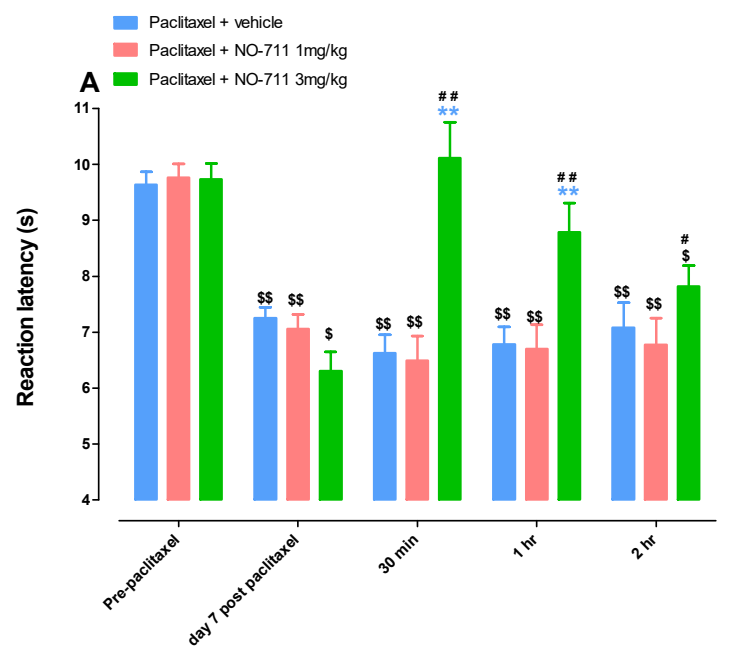

Time after drug administration

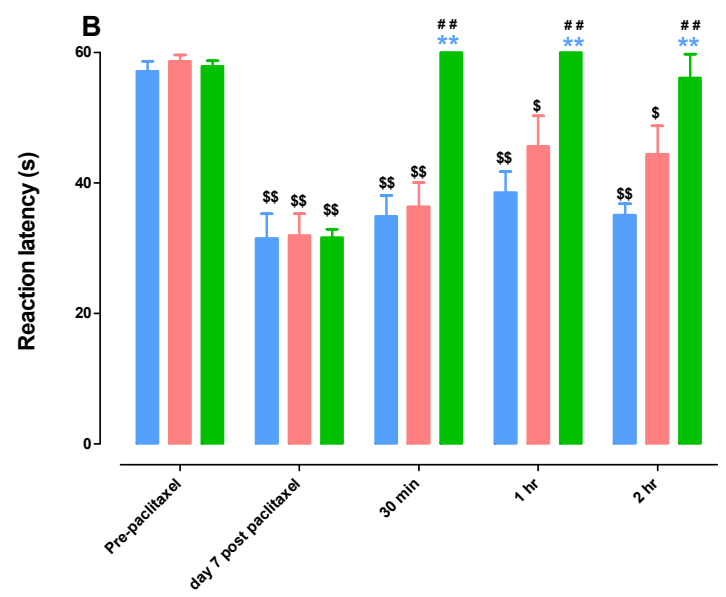

Time after drug administration 
Figure $\mathbf{5}$ (on next page)

Time course of the mean time spent on the rotarod (s) for NO-711 in a rotarod test in naïve $B A L B / c$ mice.

(A) At a constant speed of $4 \mathrm{rpm}$. Each point represents the mean \pm S.E.M of values obtained from 8 animals. (B) At an accelerating mode from 4 rpm to 40 rpm over 5 minutes. Each point represents the mean \pm S.E.M of values obtained from 12-13 animals. $* * p<0.01$ compared to drug vehicle at the same time point after treatment (two-way repeated measures ANOVA followed by Bonferroni post-test). 


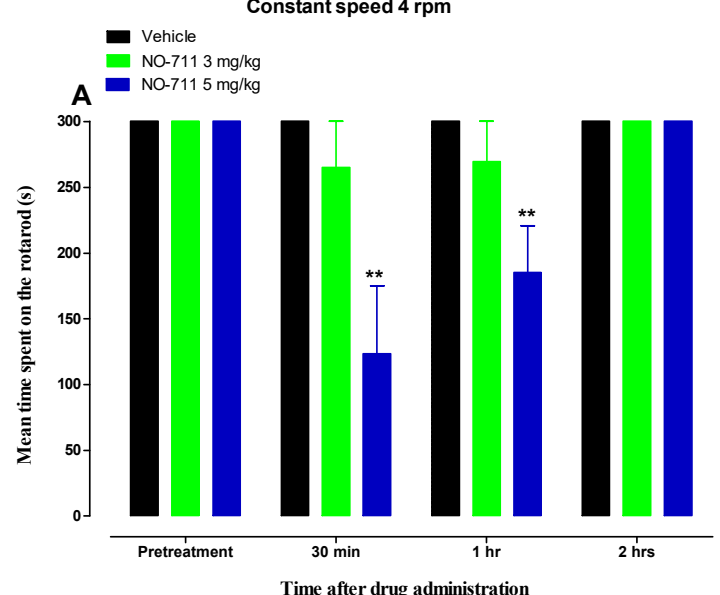

Accelerating mode $4-40 \mathrm{rpm}$ over $10 \mathrm{~min}$

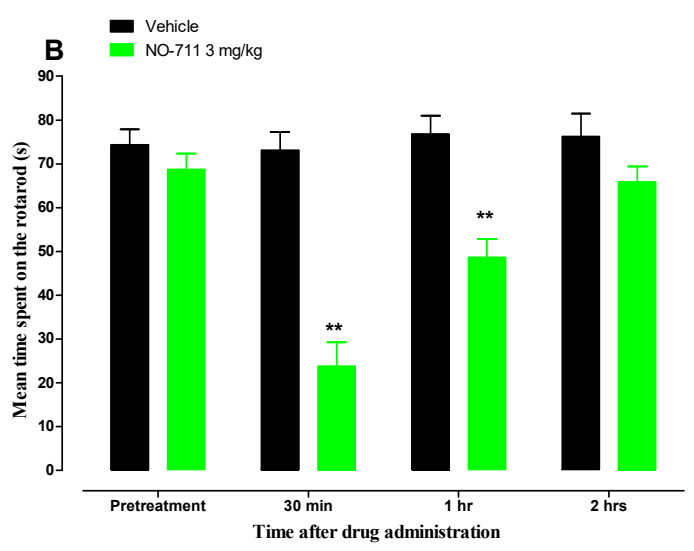

